DRAFT VERSION NOVEMBER 12, 2018

Preprint typeset using LTEX style emulateapj v. 5/2/11

\title{
ISMABS: A COMPREHENSIVE X-RAY ABSORPTION MODEL FOR THE INTERSTELLAR MEDIUM
}

\author{
E. GatuzZ ${ }^{1}$, J. García ${ }^{2}$, T.R. KAllman ${ }^{3}$, C. MendozA ${ }^{1}$, T.W. GorCZyCA ${ }^{4}$ \\ Draft version November 12, 2018
}

\begin{abstract}
We present an X-ray absorption model for the interstellar medium, to be referred to as ISMabs, that takes into account both neutral and ionized species of cosmically abundant elements, and includes the most accurate atomic data available. Using high-resolution spectra from eight X-ray binaries obtained with the Chandra High Energy Transmission Grating Spectrometer, we proceed to benchmark the atomic data in the model particularly in the neon K-edge region. Compared with previous photoabsorption models, which solely rely on neutral species, the inclusion of ions leads to improved spectral fits. Fit parameters comprise the column densities of abundant contributors that allow direct estimates of the ionization states. ISMabs is provided in the appropriate format to be implemented in widely used X-ray spectral fitting packages such as XSPEC, ISIS and SHERPA.

Subject headings: atomic data - ISM: abundances - ISM: atoms - ISM: general - X-rays: binaries - X-rays: ISM
\end{abstract}

\section{INTRODUCTION}

The physical properties of the interstellar medium (ISM) are coupled to the star life cycles and affect the dynamics of the Galaxy; therefore, the understanding of the ISM composition and phases has broad relevance. The ISM has been studied by a variety of observational techniques, e.g. $21 \mathrm{~cm}$ radio emission (Dickey \& Lockman 1990), IR (Dwek \& Arendt 1992), and UV absorption spectroscopy (Jenkins 2005). Xray high-resolution spectroscopy has lately become an attractive method as it provides data that are unique and complementary to those gathered with other techniques. By using a bright continuum-dominated X-ray source as a backlight and grating spectrometers, e.g. the Chandra High Energy Transmission Grating Spectrometer (HETGS) or the XMMNewton Reflection Grating Spectrograph (RGS), it is possible through the modeling of the observed absorption features to unravel key ISM physical and chemical conditions. Due to the penetrating power of X-rays through the gas and dust, highresolution X-ray spectroscopy allows the direct measurement of spectral features such as absorption lines and edges that lead to the identification of multiple atomic ionization species, molecules, solid composites, and to the determination of binding energies (Yao et al. 2009). Reliable templates are thus needed to diagnose the different absorption signatures in such a diverse and complex plasma.

Useful photoabsorption models to render the ISM features have been progressively refined and incorporated in the $\mathrm{X}$ ray spectral fitting packages. Wabs, a model included in the XSPEC X-ray fitting package, was based on a compilation (Morrison \& McCammon 1983) of the photoabsorption cross sections by Henke et al. (1982), and included the solar abundances of Anders \& Ebihara (1982); however, it neglected contributions from ions, molecules, grains, and abundance enhancements. Due to this latter limitation, Balucinska-Church

${ }^{1}$ Centro de Física, Instituto Venezolano de Investigaciones Científicas (IVIC), PO Box 20632, Caracas 1020A, Venezuela egatuzz@ivic.gob.ve, claudio@ivic.gob.ve

${ }^{2}$ Harvard-Smithsonian Center for Astrophysics, Cambridge, MA 02138. USA

NASA Goddard Space Flight Center, Greenbelt, MD 20771, USA

${ }^{4}$ Department of Physics, Western Michigan University, Kalamazoo, MI
\& McCammon (1992) energy fitted these cross sections for 17 elements in a model referred to as Phabs, allowing the treatment of abundances as adjustable parameters. Within the XSPEC spectral fitting package (Arnaud 1996), TBabs considered improved cross sections and revised abundances as well as contributions from grains and the $\mathrm{H}_{2}$ molecule (Wilms et al. 2000). In order to comply with Chandra and XMM-Newton spectral requirements, an updated version of this model (TBnew) has been available, which implements finer cross-section meshes to delineate the resonance structures of the oxygen and neon $\mathrm{K}$ edges and the iron $\mathrm{L}$ edge. It must be noted that all these models only consider photoabsorption by neutral atomic species. On the other hand, Pinto et al. (2010, 2013) have analyzed ISM absorption in lowmass X-ray binary (LMXB) spectra with the SPEX package using three absorption models that assume ionization and energy balance: hot for collisionally ionized plasmas; slab for plasmas with arbitrary compositions; and amol that takes into account the effects of dust and molecules. Gatuzz et al. (2013) have carried out a study of ISM oxygen K absorption using Chandra spectra of the LMXB XTE J1817-330. Spectral fits were performed with XSPEC using a physical model referred to as warmabs, which is based on precalculated tabulations of ionic fractions and level populations from the XSTAR photoionization code to generate synthetic emission and transmission spectra. In this work, a model benchmark of the theoretical atomic data for oxygen ions by García et al. (2005) required wavelength shifts for both the $\mathrm{K}$ lines and photoionization cross sections in order to fit the observed spectra accurately. Moreover, an attempt to develop a definitive absorption model for neutral oxygen with a new computed cross section revealed standing discrepancies between the laboratory and observed line positions (Gorczyca et al. 2013). The warmabs model has been subsequently used with the adjusted O I cross sections of García et al. (2005) and Gorczyca et al. (2013) to analyze ISM oxygen absorption toward eight LMXBs (Gatuzz et al.2014). Oxygen abundances and ionization parameters along different lines of sight were therein determined, finding only small abundance differences due to the theoretical atomic data, and estimating the $N(\mathrm{O}$ II $) / N(\mathrm{O})$ column-density ratio to be around $10 \%$. In the case of magnesium, new photoabsorption cross sections by 
Table 1

Chandra HETG Observations

\begin{tabular}{|c|c|c|c|c|c|c|}
\hline Source & ObsID & Date & $\begin{array}{c}\text { Exposure } \\
(\mathrm{ks})\end{array}$ & Read mode & $\begin{array}{c}\text { Galactic } \\
\text { coordinates }\end{array}$ & $\begin{array}{c}\text { Distance } \\
(\mathrm{kpc})\end{array}$ \\
\hline \multirow[t]{4}{*}{$4 U$ 1636-53 } & 105 & 1999 Oct 20 & 29 & TIMED & \multirow[t]{4}{*}{$(332.9,-4.8)$} & \multirow[t]{4}{*}{$5.95^{a}$} \\
\hline & 1939 & 2001 Mar 28 & 27 & TIMED & & \\
\hline & 6635 & 2006 Mar 22 & 23 & CONTINUOUS & & \\
\hline & 6636 & 2007 Jul 02 & 26 & CONTINUOUS & & \\
\hline \multirow[t]{3}{*}{$4 \mathrm{U} 1735-44$} & 704 & 2000 Jun 09 & 24 & TIMED & \multirow[t]{3}{*}{$(346.0,-6.9)$} & \multirow[t]{3}{*}{$6.5^{b}$} \\
\hline & 6637 & 2006 Aug 17 & 25 & CONTINUOUS & & \\
\hline & 6638 & 2007 Mar 15 & 23 & CONTINUOUS & & \\
\hline \multirow{5}{*}{ 4U 1820-30 } & 1021 & 2001 Jul 21 & 9.6 & TIMED & \multirow{5}{*}{$(2.7,-7.9)$} & \multirow{5}{*}{$7.6^{c}$} \\
\hline & 1022 & 2001 Sep 12 & 11 & TIMED & & \\
\hline & 6633 & 2006 Aug 12 & 25 & CONTINUOUS & & \\
\hline & 6634 & 2006 Oct 20 & 26 & CONTINUOUS & & \\
\hline & 7032 & 2006 Nov 05 & 47 & CONTINUOUS & & \\
\hline \multirow[t]{2}{*}{ Cygnus X-1 } & 3407 & 2001 Oct 28 & 17 & CONTINUOUS & \multirow[t]{2}{*}{$(71.3,3.0)$} & \multirow[t]{2}{*}{$7.2^{d}$} \\
\hline & 3724 & 2002 Jul 30 & 8.8 & CONTINUOUS & & \\
\hline \multirow[t]{4}{*}{ Cygnus X-2 } & 8170 & 2007 Aug 25 & 65 & CONTINUOUS & \multirow[t]{4}{*}{$(87.3,-11.3)$} & \multirow[t]{4}{*}{$2.14^{e}$} \\
\hline & 8599 & 2007 Aug 23 & 59 & CONTINUOUS & & \\
\hline & 10881 & 2009 May 12 & 66 & CONTINUOUS & & \\
\hline & 1102 & 1999 Sep 23 & 28 & TIMED & & \\
\hline \multirow[t]{2}{*}{ GX 9+9 } & 703 & 2000 Aug 22 & 20 & TIMED & \multirow[t]{2}{*}{$(8.5,9.0)$} & \multirow[t]{2}{*}{$8^{f}$} \\
\hline & 11072 & 2010 Jul 13 & 95 & TIMED & & \\
\hline Sco $X-1$ & 3505 & 2003 Jul 21 & 16 & CONTINUOUS & $(359,23.7)$ & $2.8^{g}$ \\
\hline \multirow[t]{4}{*}{ XTE J1817-330 } & 6615 & 2006 Feb 13 & 18 & CONTINUOUS & \multirow[t]{4}{*}{$(359.8,-7.9)$} & \multirow[t]{4}{*}{$1-4^{h}$} \\
\hline & 6616 & 2006 Feb 24 & 29 & CONTINUOUS & & \\
\hline & 6617 & 2006 Mar 15 & 47 & CONTINUOUS & & \\
\hline & 6618 & 2006 May 22 & 51 & CONTINUOUS & & \\
\hline \multicolumn{7}{|c|}{ 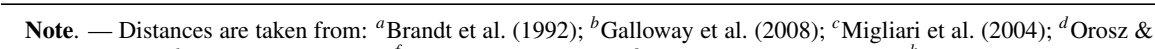 } \\
\hline Kuulkers (1999); ${ }^{\mathrm{Ma}}$ & sey et al. & $995 ;$ Zdziarski & tal. $2004 ; ;^{8}$ & Bradshaw et al. (1999 & and Sala \& Grei & er 2006). \\
\hline
\end{tabular}

Hasoğlu et al. (2014) in warmabs were used to fit the $\mathrm{Mg} \mathrm{K}$ edge in XMM-Newton spectra of the LMXB GS 1826-238 predicting that most of this element was in ionized form.

The warmabs model includes the most accurate atomic data for atomic neutrals and ions, but it is constrained by the assumption of ionization equilibrium. This may not be practical owing to deviations in the ionizing energy distribution, other ionization mechanisms, or the superposition of multiple components along the line of sight. Hence, we present a model of ISM X-ray absorption, to be referred to as ISMabs, that takes into account both neutrals and charged ions allowing their column densities to vary as free parameters. We benchmark ISMabs by means of fits to high-resolution spectra from X-ray binary sources obtained with the Chandra HETGS and comparisons with the TBnew and warmabs models. This procedure has involved a detailed study of the oxygen and neon $\mathrm{K}$ edges as well as the iron L edge. The outline of this paper is as follows. In Section 2 the data reduction process is briefly summarized, and in Section 3 we describe the selected atomic database and the X-ray absorption model developed to render the ISM absorption features. In Section 4 we show fit comparisons of the TBnew and ISMabs models. Results obtained from the broadband fit are reviewed in Section 5, with particular attention to the neon K- and iron L-edge regions, followed by a detailed discussion of the fits in Section 6. Finally, in Appendix A we give an account of the importance of ionization balance in abundance determinations.

\section{OBSERVATIONS AND DATA REDUCTION}

The most suitable sources for X-ray ISM studies are bright LMXBs as they do not usually show intrinsic spectral features, and preferably with photon fluxes with high signalto-noise ratios to enable the measurement of the ISM con- tribution. To hone the present photoabsorption model, we have selected eight candidates from the catalog of LMXBs (Liu et al. 2007) and the Transmission Grating Catalog and Archive (TGCat) ${ }^{5}$ such that the total number of counts in each HETGS spectrum is greater than $10^{6}$. We have also included spectra from the high-mass X-ray binary Cygnus X-1 due to its high photon flux, giving a total of 25 observations. Observational details are listed in Table 1, where it is worth adding that the high-energy resolution of the Chandra gratings is certainly indispensable in the present modeling developments. The spectra have been reduced with the standard CIAO threads 6 , the zero-order position being estimated in each case with the findzo algorithm 7 Spectral fitting was carried out with the XSPEC package (version 12.8.1 8 .

\section{ISMABS PHOTOABSORPTION MODEL}

ISM photoabsorption causes significant modifications to the observed X-ray spectra, and must therefore be taken into account in any high-resolution spectroscopic analysis. For this purpose, Wilms et al. (2000) developed the TBabs and TBnew absorption models that have been widely used in the last decade. However, they only include neutral atomic species compromising their ability to render complex ISM spectral structures such as the $\mathrm{O}, \mathrm{Ne}$, and $\mathrm{Mg} \mathrm{K}$ edges (Juett et al. 2004, 2006, Hasoğlu et al. 2014). Given this limitation, we have devised a new ISM X-ray absorption model-to be referred to as ISMabs - that compiles the most accurate atomic data available for neutrally, singly, and doubly ionized species of $\mathrm{H}, \mathrm{He}, \mathrm{C}, \mathrm{N}, \mathrm{O}, \mathrm{Ne}, \mathrm{Mg}, \mathrm{Si}, \mathrm{S}, \mathrm{Ar}$ and $\mathrm{Ca}$; in the case of iron, an experimental cross section has been chosen to

\footnotetext{
${ }^{5}$ http://tgcat.mit.edu/

${ }^{6} \mathrm{http}: / / \mathrm{cxc}$. harvard.edu/ciao/threads/gspec.html

${ }^{7} \mathrm{http}: / /$ space.mit.edu/cxc/analysis/findzo/

${ }^{8} \mathrm{http}: / /$ heasarc.nasa.gov/xanadu/xspec/
} 
Table 2

Cross-sections included on ISMabs

\begin{tabular}{|c|c|c|c|}
\hline Ion & Source & Ion & Source \\
\hline $\begin{array}{l}\text { H } \\
\text { He I } \\
\text { He II } \\
\text { C I, C II, C III } \\
\text { N I, N II,N III } \\
\text { O I } \\
\text { O II, O III } \\
\text { Ne I } \\
\text { Ne II, Ne III }\end{array}$ & 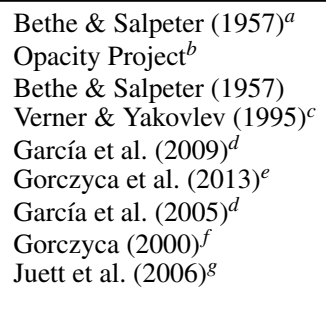 & $\begin{array}{l}\text { Mg I, Mg II, Mg III } \\
\text { Si I } \\
\text { Si II, Si III } \\
\text { S I } \\
\text { S II, S III } \\
\text { Ar I } \\
\text { Ar II, Ar III } \\
\text { Ca I } \\
\text { Ca II, Ca III } \\
\text { Fe }\end{array}$ & \begin{tabular}{|l|} 
Hasoğlu et al. $(2014)^{c}$ \\
Verner \& Yakovlev \\
Witthoeft et al. $(2009)^{a}$ \\
Verner \& Yakovlev \\
Witthoeft et al. $(2009)$ \\
Verner \& Yakovlev \\
Witthoeft et al. $(2011)^{a}$ \\
Verner \& Yakovlev \\
Witthoeft et al. (2019) \\
Kortright \& Kim 2000$)^{h}$ \\
\end{tabular} \\
\hline
\end{tabular}

\footnotetext{
a Analytic method.

b Calculated with the $R$-matrix method.

${ }^{\mathrm{c}}$ Semiempirical fitting formula based on Hartree-Dirac-Slater computations.

${ }^{\mathrm{d}}$ Calculated with the Breit-Pauli $R$-matrix method.

e Derived from an analytical formula.

${ }^{\mathrm{f}}$ Based on $R$-matrix calculations and an optical potential.

$\mathrm{g}$ Based on the calculation by Gorczyca 2000 and adjusted to astrophysical observations.

${ }^{\mathrm{h}}$ Experimental measurements of metallic iron.
}

model the L-edge absorption region (see Section 3.1.1).

Photoabsorption in ISMabs takes the form

$$
I_{\text {obs }}(E)=I_{\text {source }}(E) \exp (-\tau)
$$

where $I_{\text {obs }}(E)$ is the observed spectrum, $I_{\text {source }}(E)$ the spectrum emitted by the source, and $\exp (-\tau)$ is the absorption coefficient. The optical depth $\tau$ is usually defined as

$$
\tau=\sigma_{\text {gas }} N(\mathrm{H}),
$$

$\sigma_{\text {gas }}$ being the total photoionization cross section comprising all the atoms and $N(\mathrm{H})$ is the hydrogen column density. If a neutral gas is considered, $\sigma_{\text {gas }}$ may be written

$$
\sigma_{\text {gas }}=\sum_{Z} A_{Z} \times \sigma_{Z}
$$

where $\sigma_{Z}$ is the photoionization cross section of a chemical element with atomic number $Z$, and $A_{Z}$ gives its abundance relative to hydrogen. It is worth noting that, due to the abundance dependency on column density, there is a degeneracy between $A_{Z}$ and $N(\mathrm{H})$, making it possible by varying one or the other to obtain multiple solutions in the calculation of $\tau$. Consequently, we prefer to adopt the following definition for $\tau:$

$$
\tau=\sum_{i}^{k} \sigma_{i}(E) N(i)
$$

here $\sigma_{i}(E)$ and $N(i)$ are respectively the cross section and column density for the $i$-th ion. We therefore manage the ion column densities, including $N(\mathrm{H})$, as model parameters in order to determine $\tau$, thus avoiding any parametric ambivalence. I SMabs always assumes that $N(\mathrm{He} \mathrm{I})=N(\mathrm{H} \mathrm{I}) / 10$, and as a default, $N(\mathrm{He}$ II $)=0$. But $N(\mathrm{He}$ II $)$ is a free parameter which the user can modify if desired. It must be emphasized that there are no changes to the cross sections during fitting except for interpolation; i.e. they are read just once by the code when the model is invoked. The energy range covered by the model is $0.1-10 \mathrm{keV}$. The redshift $z$ is also a model parameter, and we will include turbulent broadening in the next revision to ISMabs (with the turbulence velocity $v$ as the model parameter in units of $\mathrm{km} \mathrm{s}^{-1}$ ); however, observations point to a turbulence $\left(v<100 \mathrm{~km} \mathrm{~s}^{-1}\right)$ that cannot be detected by Chandra (Gatuzz et al. 2013). Furthermore, for this velocity range, the natural broadening of inner-shell resonances dominate over turbulence broadening. ISMabs has been specially developed for X-ray fitting packages such as XSPEC (Arnaud 1996), ISIS (Houck \& Denicola 2000) and SHERPA (Freeman et al. 2001).

\subsection{Atomic data}

In addition to high signal-to-noise X-ray spectra from a back-light source, reliable atomic data are required to model the ISM absorption signatures. The atomic databases used by many of the currently available X-ray absorption models are not complete and of sufficient accuracy to model gratingresolution spectra. This situation was illustrated in the work of Gatuzz et al. (2013), who used four high-resolution Chandra spectra of the LMXB XTE J1817-330 to benchmark the oxygen theoretical atomic database (García et al. 2005) in the XSTAR photoionization code. The photoabsorption cross sections of the lowly ionized species required several adjustments: wavelength shifts of the $\mathrm{K} \alpha$ resonances in $\mathrm{O}$ I (29 $\mathrm{m \AA})$ and O II (75 $\mathrm{m} \AA)$ and of the whole cross sections for both of these ions. Moreover, a new and more accurate theoretical cross section for O I by Gorczyca et al. (2013) showed significant differences in the oscillator-strength distribution, and brought to light a discrepancy in the wavelength scale, namely $33 \mathrm{m \AA}$, between the observed $\mathrm{K} \alpha$ line position and the laboratory measurement (Stolte et al. 1997). The quantitative differences in photoabsorption modeling brought about by these two O I cross sections, i.e. García et al. (2005) and Gorczyca et al. (2013), were examined by Gatuzz et al.(2014) in a study of ISM absorption along several lines of sight, constraining them to less than $5 \%$ in the average ionization parameter and oxygen abundance. A further point of interest is the presence of condensed matter in the ISM, which can be detected through the analysis of X-ray absorption fine structure in the form of spectral modulations near the photoabsorption edges, and by the absence of strong inner-shell resonance transitions below the ionization threshold (Lee \& Ravel 2005; Lee et al. 2009); however, their proper characterization would require beforehand the mastering of the atomic absorp- 

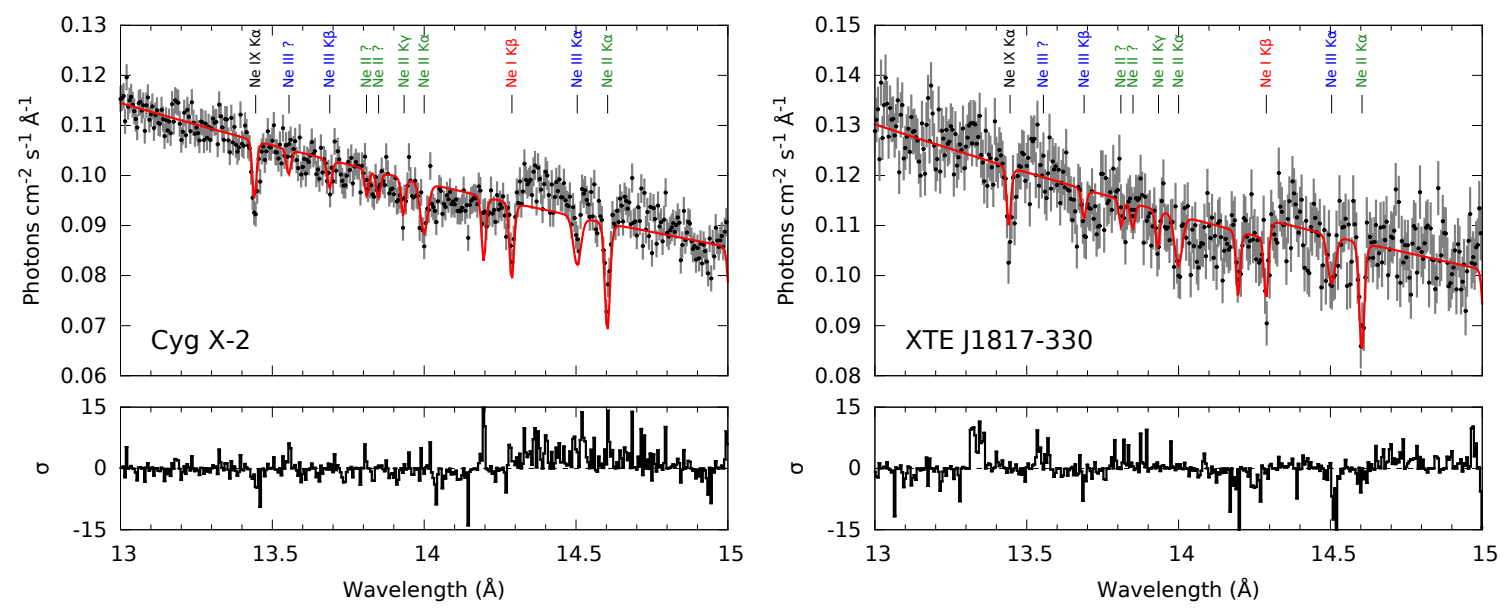

Figure 1. Chandra MEG flux spectra of the X-ray binaries Cygnus X-2 (left panel) and XTE J1817-330 (right panel) simultaneously fitted in the 13-15 $\AA$ region using a simple functional model (a power law and several Gaussian profiles). The black data points correspond to the observations while the red solid lines represent the model best fit. The bottom panels show fit residuals in units of $\sigma$.

tion edges which is the central topic of the present work.

\subsubsection{Database}

In Table 2 we list the reference sources for the cross sections incorporated in ISMabs. We include the theoretical atomic photoabsorption data for N I-N III by García et al. (2009); and by Witthoeft et al. (2009, 2011) for the singly and doubly ionized species of $\mathrm{Si}, \mathrm{S}, \mathrm{Ar}$, and $\mathrm{Ca}$, which were calculated with the $R$-matrix method using an optical potential to account for Auger damping. For the neutral species of the latter four elements, we adopt, in the absence of better data, the theoretical cross sections of Verner \& Yakovlev (1995) even though they do not take into account the inner-shell resonances that give rise to the $\mathrm{K}$-edge structures. These cross sections are also used for the neutral, singly, and doubly ionized species of C. For oxygen ions, we select the theoretical cross section for O I by Gorczyca et al. (2013) and those by García et al. (2005) for O II and O III, including the corrections specified by Gatuzz et al. (2013) as previously mentioned. In the case of neon, we take the $R$-matrix data (including Auger damping) by Gorczyca (2000) for Ne I and by Juett et al. (2006) for $\mathrm{Ne}$ II and Ne III. For the magnesium ions, Mg I-Mg III, we take the photoabsorption cross sections computed by Hasoğlu et al. (2014) with the $R$-matrix method that account for orbital relaxation effects to reduce a previous over-estimate of the K-edge position (Witthoeft et al. 2009, 2011).

In the case of iron, Juett et al. (2006) fitted the L-edge region using the experimental cross section of metallic iron by Kortright \& Kim (2000). On the other hand, Pinto et al. (2010 2013) adjusted the L edge using the atomic data for the neutral by Verner \& Yakovlev (1995), but included a molecular solid component since the edge cannot be fitted with a pure gas model. Using Chandra HETG spectra from five X-ray binaries, Miller et al. (2009) adjusted the iron L edge in the TBnew absorption model with the experimental cross section (Kortright \& Kim 2000). In the present work, we adopt the metallic iron measurements (Kortright \& Kim 2000) but with a wavelength correction of $+39 \mathrm{~mA}$. This shift was originally suggested by Juett et al. (2006), who argued that the energy resolution of the laboratory data in combination with the HETG absolute instrumental wavelength accuracy would allow it. We also considered the widely used atomic Fe L-shell cross section of Verner \& Yakovlev (1995), but the result- ing spectral fit was inferior to that obtained with the shifted cross section of Kortright \& Kim (2000). Finally, it is worth pointing out that both warmabs and ISMabs use the same atomic database except for $\mathrm{Fe}$ since warmabs uses the $\mathrm{Fe}$ cross-section of Verner \& Yakovlev (1995).

\subsubsection{Ne benchmark}

Following the benchmark of the oxygen cross sections by Gatuzz et al. (2013), we proceed to compare the theoretical and observed absorption lines in the Ne-edge region of spectra with high signal-to-noise fluxes, namely Cygnus $\mathrm{X}-2$ and XTE J1817-330. Figure 1 shows the best fits in the 13-15 interval using a simple functional model, i.e. a powerlaw and several Gaussian profiles. For each source, all observations are fitted simultaneously, but the spectra are combined for illustrative purposes; the black data points correspond to the observations while the red solid lines represent the model best fit for each case. The bottom panels show fit residuals in units of $\sigma$. Several absorption lines are clearly observed including those due to the $\mathrm{K} \alpha$ transitions of Ne II and Ne III. Identifications of the spectral features and line wavelength positions are listed in Table 3 where the estimates by Juett et al. (2004), Yao et al. (2009), and Liao et al. (2013) are also tabulated for comparison. We have identified absorption lines due to transitions in Ne I, Ne II, and Ne III, and the line at $14.195 \pm 0.005 \AA$ presumably belongs to the $\mathrm{K} \alpha$ transition of $\mathrm{Ne} \mathrm{V}$. A comparison between the theoretical resonance positions with those determined from the spectral fits reveal the inaccuracies of the theoretical atomic data.

The left panel of Figure 2 shows with solid lines the theoretical cross sections for Ne I-Ne III (Gorczyca 2000, Juett et al. 2006) that are included in the ISMabs model. The vertical dashed lines are placed at the positions of the absorption features determined from the functional fits. In Table 3 we give the line assignments based on a comparison between our functional fits and the theoretical atomic data. Identifications made in previous work using astrophysical data are also listed: Cygnus X-2 was analyzed by Juett et al. (2006) and Yao et al.(2009) while XTE J1817-330 was included by Liao et al. (2013) in their analysis of 11 LMXBs. It may be noted that the Ne III $\mathrm{K} \alpha$ position as well as those with $n>2$ are displaced with respect to the observations, and the cross sections of $\mathrm{Ne} \mathrm{I}$ and $\mathrm{Ne}$ II also show similar displacements. In this re- 

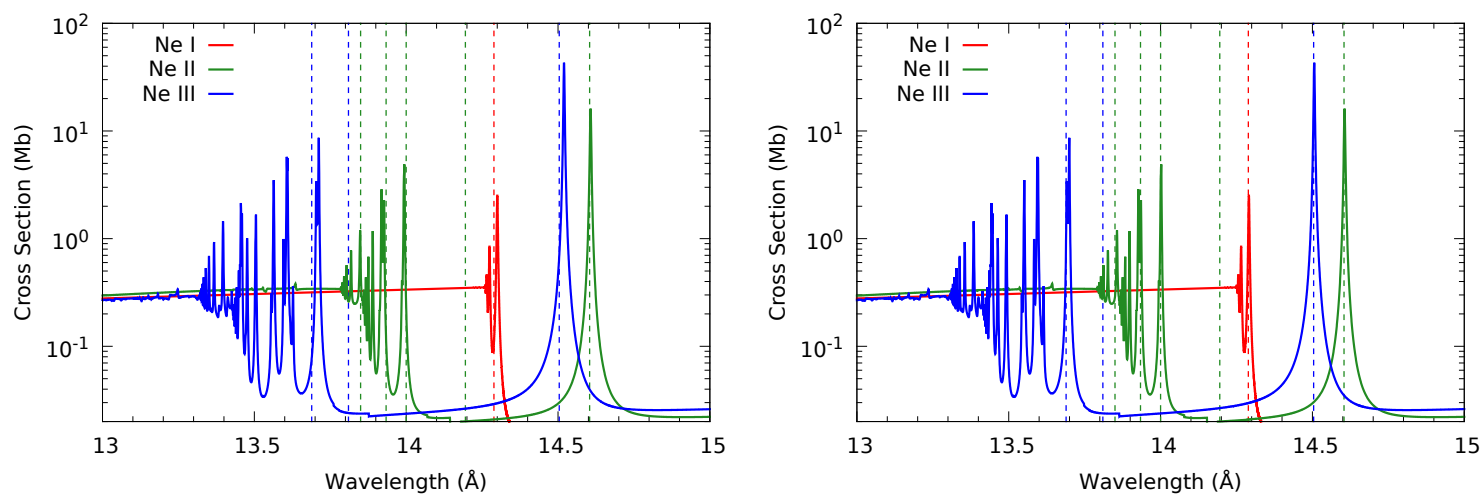

Figure 2. Theoretical photoabsorption cross sections for Ne I, Ne II, and Ne III (Gorczyca 2000, Juett et al. 2006). The vertical dashed lines are placed at the wavelengths of the observed absorption lines. The left panel displays the original cross sections while the right shows them after the wavelength shifts have been applied.

Table 3

Ne Absorption Line Assignments

\begin{tabular}{|c|c|c|c|c|c|c|}
\hline Ion & Transition & $\begin{array}{l}\lambda^{a} \\
(\AA)\end{array}$ & $\begin{array}{l}\lambda^{b} \\
(\AA)\end{array}$ & $\begin{array}{l}\lambda^{c} \\
(\AA)\end{array}$ & $\begin{array}{l}\lambda^{d} \\
(\AA)\end{array}$ & $\begin{array}{l}\lambda^{e} \\
(\AA)\end{array}$ \\
\hline $\mathrm{Ne}$ III & $\mathrm{K} \gamma$ & 13.453 & $13.441 \pm 0.001$ & $13.446 \pm 0.007(\mathrm{Ne}$ IX K $\alpha)$ & $13.444 \pm 0.001$ & $13.445 \pm 0.001$ \\
\hline $\mathrm{Ne}$ III & $?$ & & $13.587 \pm 0.007$ & & & \\
\hline Ne III & $\mathrm{K} \beta$ & 13.709 & $13.689 \pm 0.020$ & $13.695_{-0.002}^{+0.001}$ & & $13.690_{-0.001}^{+0.006}$ \\
\hline $\mathrm{Ne}$ II & ? & & $13.810 \pm 0.001$ & $13.828 \pm 0.003(\mathrm{Ne}$ VII $\mathrm{K} \alpha)$ & & \\
\hline $\mathrm{Ne}$ II & ? & & $13.850 \pm 0.001$ & & & \\
\hline $\mathrm{Ne}$ II & $\mathrm{K} \gamma$ & $?$ & $13.934 \pm 0.002$ & $13.936_{-0.003}^{+0.002}$ & & \\
\hline \multirow[t]{2}{*}{$\mathrm{Ne}$ II } & $\mathrm{K} \beta$ & 13.992 & $14.000 \pm 0.002$ & $14.004 \pm 0.001$ & & $14.001_{-0.001}^{+0.002}$ \\
\hline & & & $14.195 \pm 0.005$ & $14.212_{-0.029}^{+0.011}(\mathrm{Ne} \vee \mathrm{K} \alpha)$ & & \\
\hline $\mathrm{Ne} I$ & $\mathrm{~K} \beta$ & 14.298 & $14.289 \pm 0.001$ & $14.295_{-0.002}^{+0.001}$ & $14.295 \pm 0.003$ & $14.294 \pm 0.001$ \\
\hline Ne III & $\mathrm{K} \alpha$ & 14.516 & $14.518 \pm 0.001$ & $14.508 \pm 0.009$ & $14.508 \pm 0.002$ & $14.507 \pm 0.002$ \\
\hline $\mathrm{Ne}$ II & $\mathrm{K} \alpha$ & 14.605 & $14.605 \pm 0.002$ & $14.608 \pm 0.007$ & $14.608 \pm 0.002$ & $14.605 \pm 0.001$ \\
\hline
\end{tabular}

a Gorczyca 2000 , and Juett et al. 2006 .

b Simultaneous functional fits with Gaussian profiles for Cygnus X-2 and XTE J1817-330.

c Liao et al. (2013).

d Juett et al. (2004).

e Yao et al. (2009).

spect, Juett et al. (2006) have previously indicated a similar wavelength offset using high-resolution Chandra spectra. In what follows we have adjusted the $\mathrm{K} \alpha$ resonance positions, and shifted the cross sections for the continuum and higher resonances for these three species in order to obtain the best possible agreement with the observed lines. The shifts are, respectively, $-3.2 \mathrm{~m} \AA$ and $-15.7 \mathrm{~m} \AA$ for the $\mathrm{Ne}$ II and $\mathrm{Ne}$ III $\mathrm{K} \alpha$ resonances; and $-11 \mathrm{~m} \AA,+7.6 \mathrm{~m} \AA$, and $-14.7 \mathrm{~m} \AA$ for the Ne I, Ne II and Ne III cross sections. The right panel of Figure 2 shows the cross sections after the application of the wavelength corrections to be finally included in the ISMabs model.

\section{TBNEW VS. ISMABS}

In this section we compare two different $\mathrm{X}$-ray absorption models, namely TBnew and ISMabs, the former including photoionization cross sections for elements with atomic number $1 \leq Z \leq 28$ (Wilms et al.2000) and the contribution from molecules and interstellar grains. It therefore favors the modeling of the observed extinction and depletions in the diffuse ISM. Model parameters are the hydrogen column density $\left(N(\mathrm{H})\right.$ in units of $\left.\mathrm{cm}^{-2}\right)$, elemental abundances $\left(A_{\mathrm{x}}\right.$ relative to solar), and the redshift $(z)$. However, TBnew only takes into account neutral atomic species, and therefore, the warm or ionized ISM phases cannot be adequately modeled. The
Table 4

Model Fits For Neutrals

\begin{tabular}{lcc}
\hline \hline Parameter & TBnew & ISMabs \\
& & \\
\hline$N(\mathrm{H})$ & $1.28 \pm 0.16$ & $1.48 \pm 0.31$ \\
$N(\mathrm{O} \mathrm{I})$ & $5.38 \pm 3.60$ & $8.65 \pm 3.21$ \\
$N(\mathrm{Ne} \mathrm{I})$ & $1.56 \pm 0.42$ & $1.63 \pm 0.14$ \\
$N(\mathrm{Fe} \mathrm{I})$ & $0.32 \pm 0.22$ & $0.54 \pm 0.24$ \\
Reduced $\chi^{2}$ & 2.07 & 1.78 \\
\multicolumn{3}{c}{} \\
\hline \multicolumn{2}{c}{ Note. $-N(\mathrm{H})$ in units of $10^{21} \mathrm{~cm}^{-2}$. Metal } \\
column densities in units of $10^{17} \mathrm{~cm}^{-2}$.
\end{tabular}

ISMabs model has been described in detail in Section 3

Figure 3 shows the Ne K-, Fe L-, and $\mathrm{O}$ K-edge regions in the broadband fit of XTE J1817-330 with both TBnew and ISMabs. In each panel, the black data points correspond to the observations while the solid lines represent the best fits with TBnew (blue line) and ISMabs (red line). The bottom panels depict fit residuals in units of $\sigma$. It is clear that the ISMabs residuals are smaller particulary around the neon and oxygen edges. In the Fe L-edge region the residuals are similar since both models use the same experimental atomic data, although small differences are noted perhaps due to the 

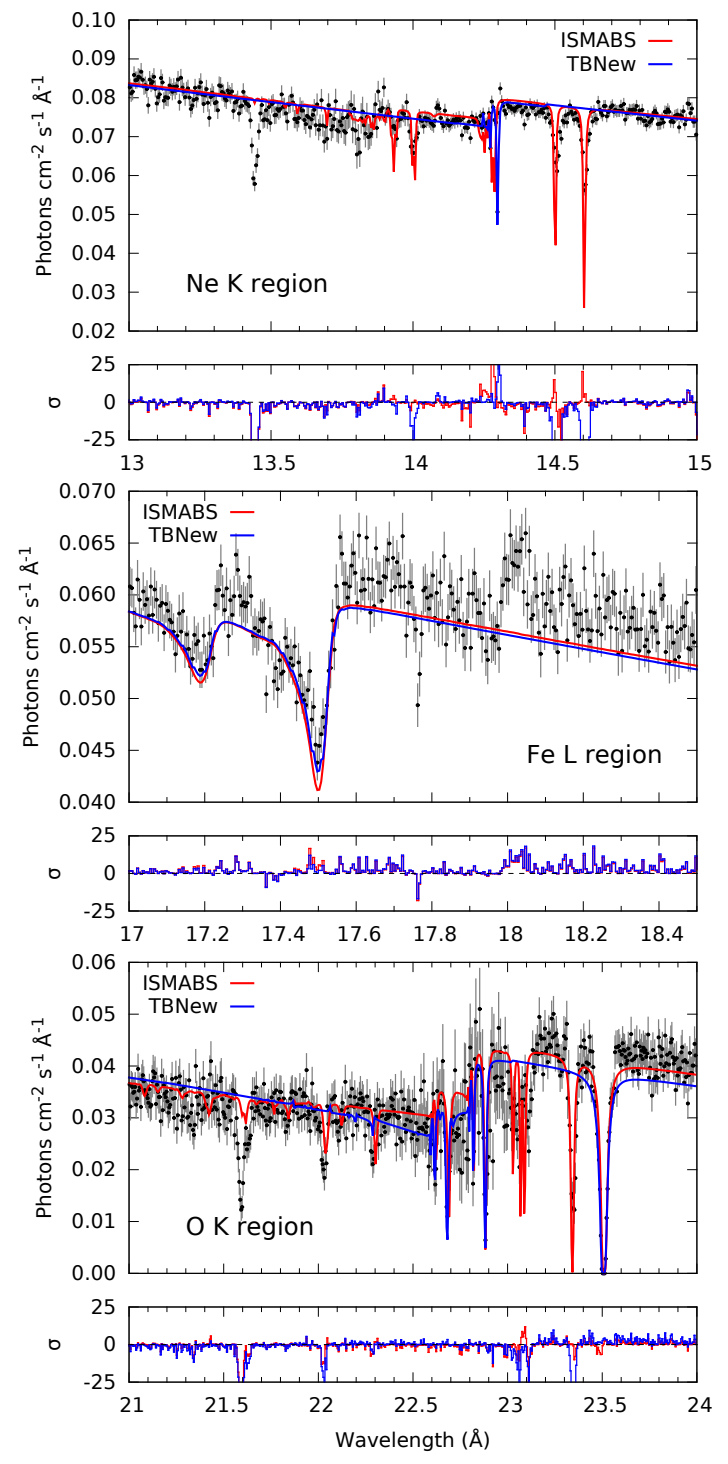

Figure 3. Spectral fits of XTE J1817-330 using the ISMabs and TBnew photoabsorption models. The black data points correspond to the observations while the solid lines represent the best fits with TBnew (blue line) and ISMbs (red line). The bottom panels depict fit residuals in units of $\sigma$. The top, middle, and bottom panels show the Ne K-, Fe L-, and O K-edge regions, respectively.

continuum. It is found that the inclusion of charged ions in addition to neutrals leads to a significant improvement in the fits. The difference in the statistics is of $\Delta \chi^{2}=12704$ if the fit is performed with ISMabs, with 4 new free parameters. Moreover, as shown in Table 4, if only the column densities for neutrals are considered (i.e. considering the same number of free parameters), both models give similar results, however the ISMabs model performs a better fit, with $\Delta \chi^{2}=1559$, due to the higher accuracy in the atomic data included.

\section{BROADBAND FIT}

Following Gatuzz et al. (2013), we have performed a broadband fit (11-24 $\AA$ ) of all the spectra listed in Table 1 using I SMabs and a power-law model. We rebin the data to at least 20 counts per channel in order to use $\chi^{2}$ statistics. For each source, all observations are fitted simultaneously varying the power-law normalization and photon index, thus accounting for possible continuum variations between observations that are always detected. The $\mathrm{H}, \mathrm{O}, \mathrm{Ne}$, and Fe column densities, as well as those of $\mathrm{O}$ and $\mathrm{Ne}$ ions, are taken as free parameters in ISMabs.

Figures 4 and 5 show the best ISMabs fits in the 11-24 interval. In the case of Sco X-1 the fit was performed in the $15-24 \AA$ interval as there are no counts below $\sim 15 \AA$. For each source the observations have been combined for illustrative purposes. It must be noted that, even though this figure looks similar to Figure 1 of Gatuzz et al. (2014), it is now obtained with our new photoabsorption model. Table 6lists the fit results where it may be appreciated that ISMabs yields the ion column densities directly, and a statistically acceptable fit is obtained for each source.

A comparison of the present hydrogen column densities with previous results is given in Table 5 finding satisfactory agreement, i.e. within the error bars. As mentioned in Gatuzz et al. (2014), the column-density variations around Cygnus X-1 are not resolved in the $21 \mathrm{~cm}$ survey; therefore, the values derived by means of spectral fits should be more reliable.

A zoom of the broadband fit in the interval $13-15 \AA$ is depicted in Figures 6 and 7 (Sco X-1 is excluded for lack of counts below $\sim 15 \AA$ ). It may be seen that the absorption features in this wavelength region are more complex than a simple edge: $\mathrm{K} \alpha$ absorption lines of Ne II and Ne II are detected at $14.60 \AA$ and $14.51 \AA$, respectively, as well as $\mathrm{K} \beta$ from Ne I, Ne II, and Ne III (14.30 $\AA, 14.00 \AA$, and $13.68 \AA$, respectively). The line at $13.45 \AA$, attributed to $\mathrm{Ne}$ III $\mathrm{K} \gamma$, is observed in all sources. Therefore, the occurrence of such lines justifies the inclusion of accurate cross sections for Ne II and Ne III in ISMabs in order to procure reliable spectral fits; for instance, in Figures 6 and 7 low residuals are found around the Ne II $\mathrm{K} \beta$ and $\mathrm{K} \gamma$ lines at $14.00 \AA$ and $13.93 \AA$, respectively. Furthermore, a similar zoom is shown in Figures 8 and 9 of the Fe L edge (17-18.5 $\AA$ ) which benchmarks the Fe I cross section adopted in ISMabs (see Section 3.1.1). It is worth recalling that the selected cross section for Fe I was obtained from measurements of solid iron, and it seems to fit the observations adequately. This finding supports previous assertions that most of the Fe in the ISM is in solid form (Lee et al. 2009).

\section{DISCUSSION AND CONCLUSIONS}

We have developed a new spectral model, referred to as ISMabs, to fit ISM X-ray absorption that takes into account photoabsorption cross sections for neutrally, singly, and doubly ionized species of cosmically abundant elements, namely $\mathrm{H}, \mathrm{He}, \mathrm{C}, \mathrm{N}, \mathrm{O}, \mathrm{Ne}, \mathrm{Mg}, \mathrm{Si}, \mathrm{S}, \mathrm{Ar}, \mathrm{Ca}$, and $\mathrm{Fe}$. In other words, ISMabs enables the determination of the ion column densities directly. Although the predominant component of the ISM is the neutral gas, the inclusion of singly and doubly ionized species provides improved fits when compared with a model (e.g. TBnew) that only accounts for neutral absorption. This shows that the understanding of the relevant atomic systems and processes, as well as the accuracy and completeness of the relevant atomic data, are both crucial to avoid incorrect interpretations of the absorption structures detected in X-ray high resolution spectra. This may occur in studies of ISM X-ray absorption that rely on fits with Gaussian profiles. $\quad$ Figure 10 shows for all sources a comparison of the oxygen, neon, and iron total column densities obtained from the ISMabs broadband fit with those derived 

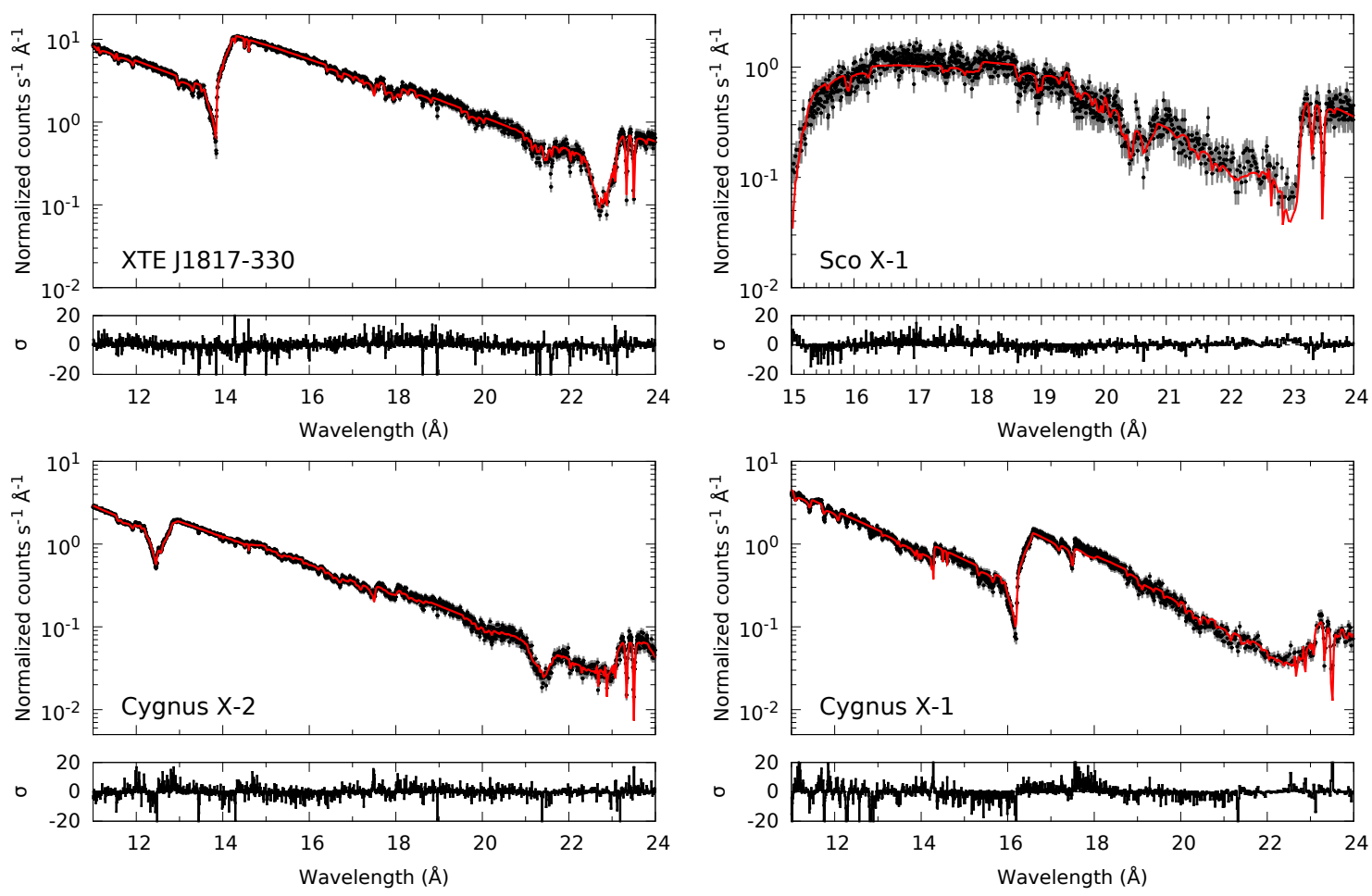

Figure 4. ISMabs best broadband fits (solid red lines) for each X-ray source included in the present study. For each source the observations have been combined for illustrative purposes.
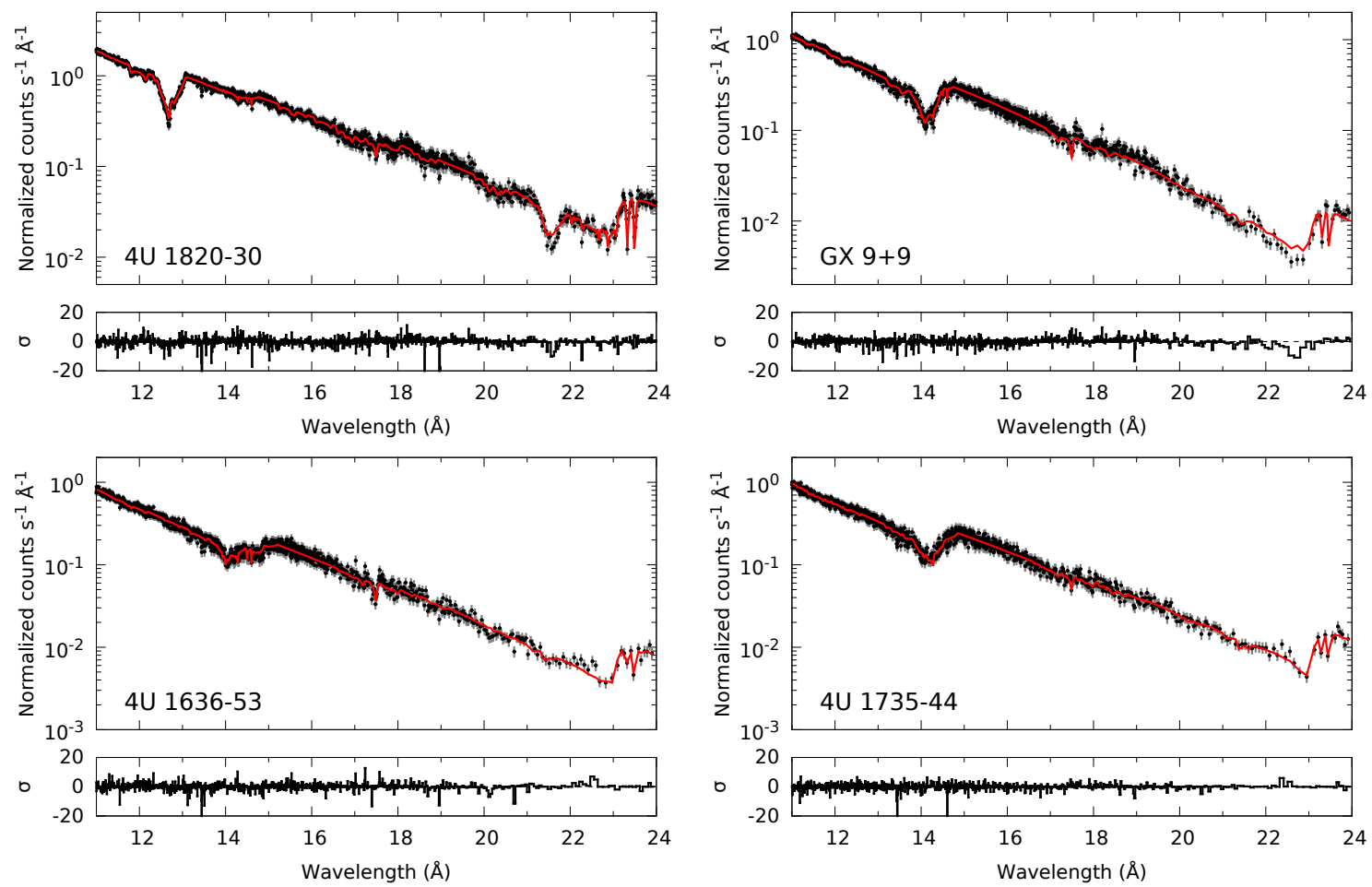

Figure 5. ISMabs best broadband fits (solid red lines) for each X-ray source included in the present study. For each source the observations have been combined for illustrative purposes. 

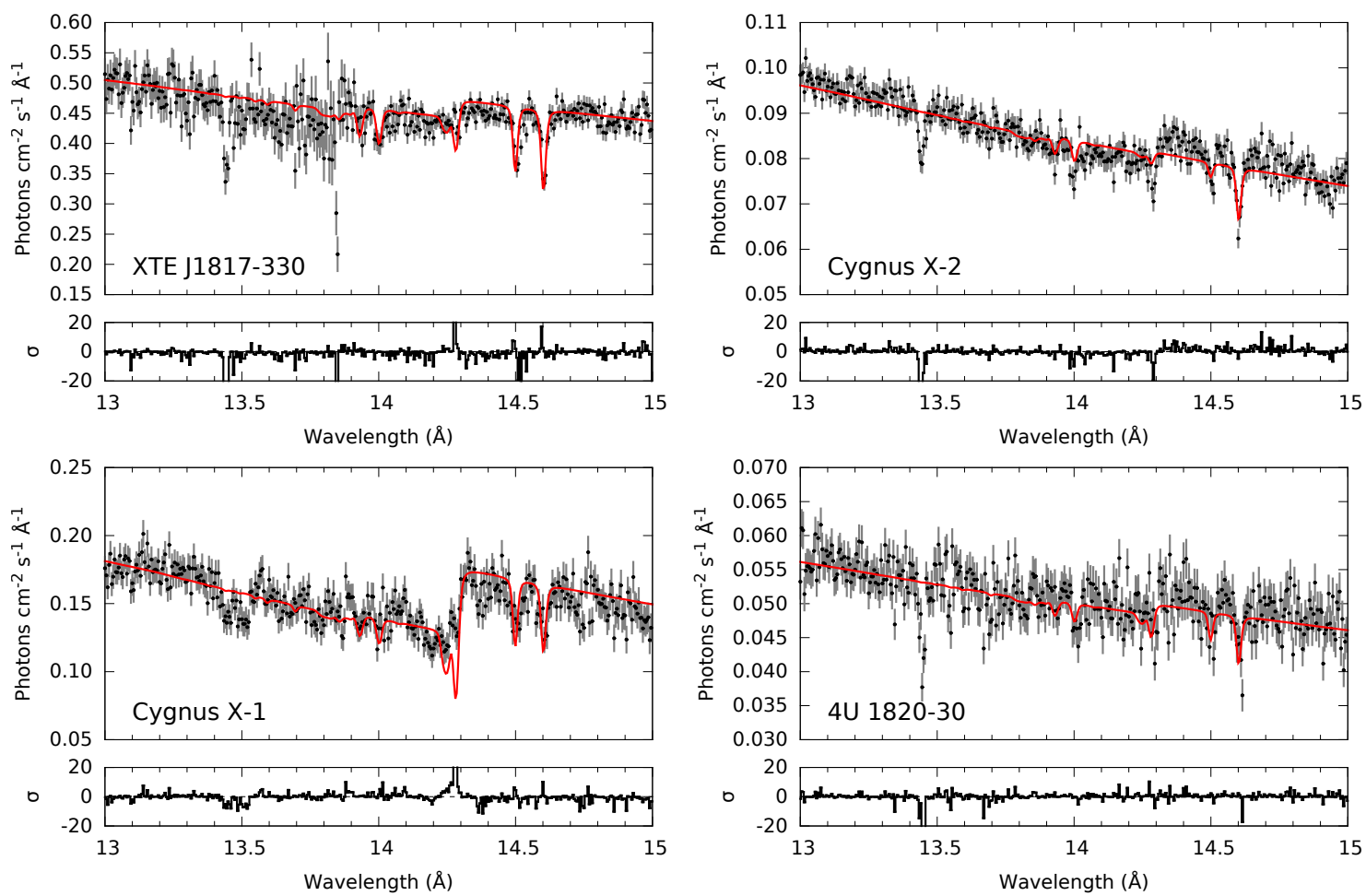

Figure 6. ISMabs best broadband fits (solid red lines) in the Ne K-edge region. For each source the observations have been combined for illustrative purposes.
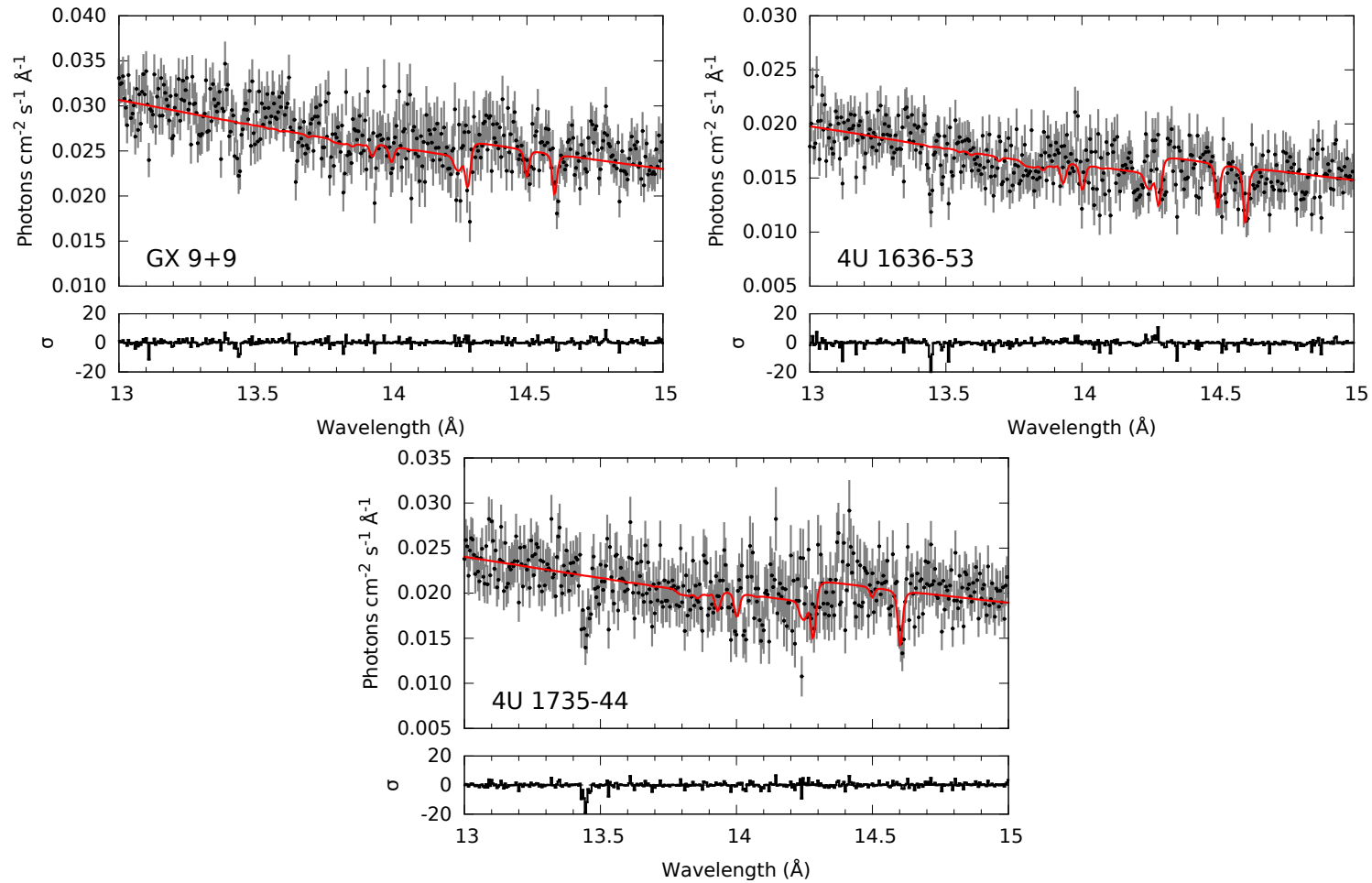

Figure 7. ISMabs best broadband fits (solid red lines) in the Ne K-edge region. For each source the observations have been combined for illustrative purposes. 

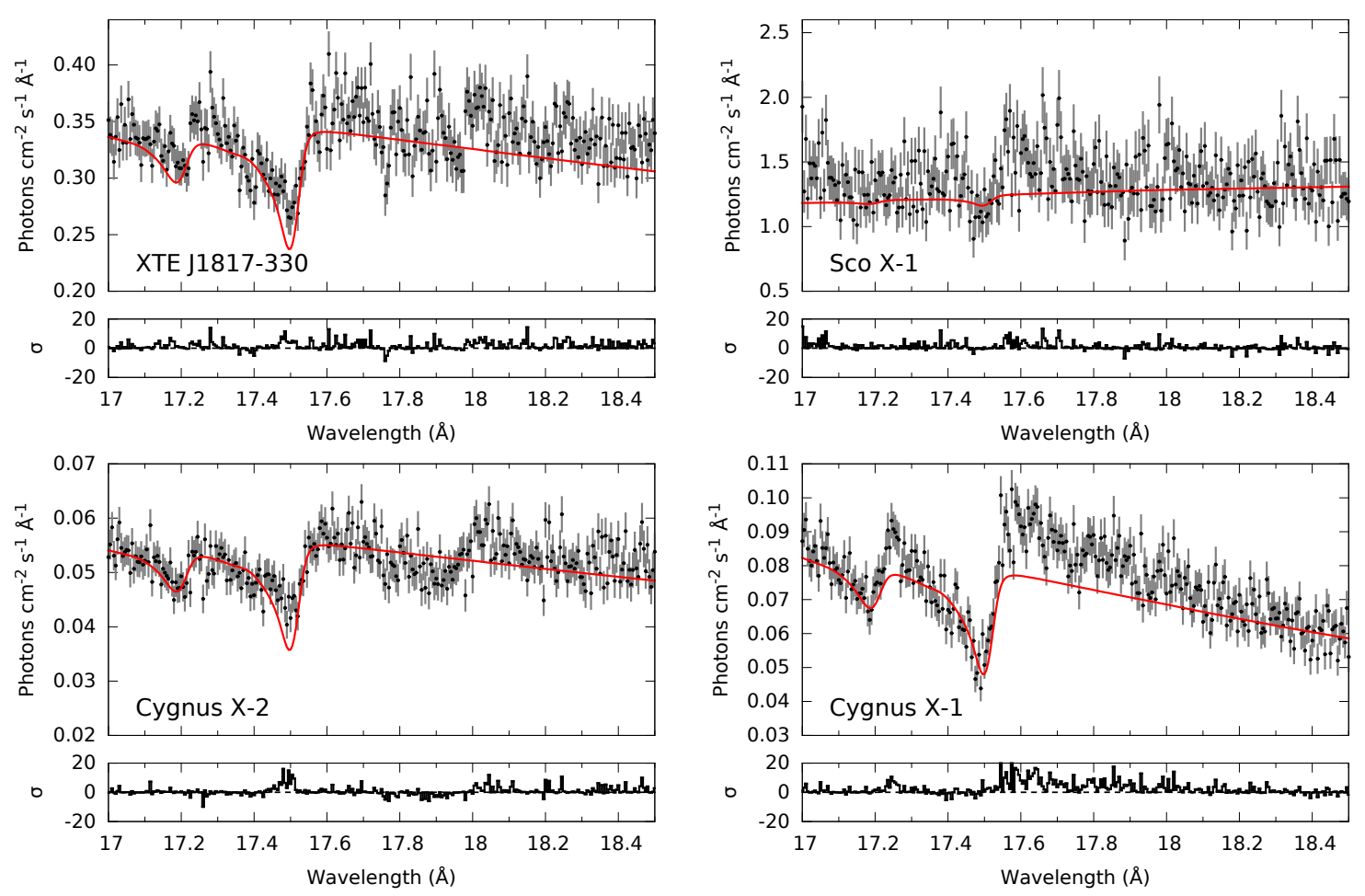

Figure 8. ISMabs best broadband fit (solid red lines) in the Fe L-edge region. For each source the observations have been combined for illustrative purposes.
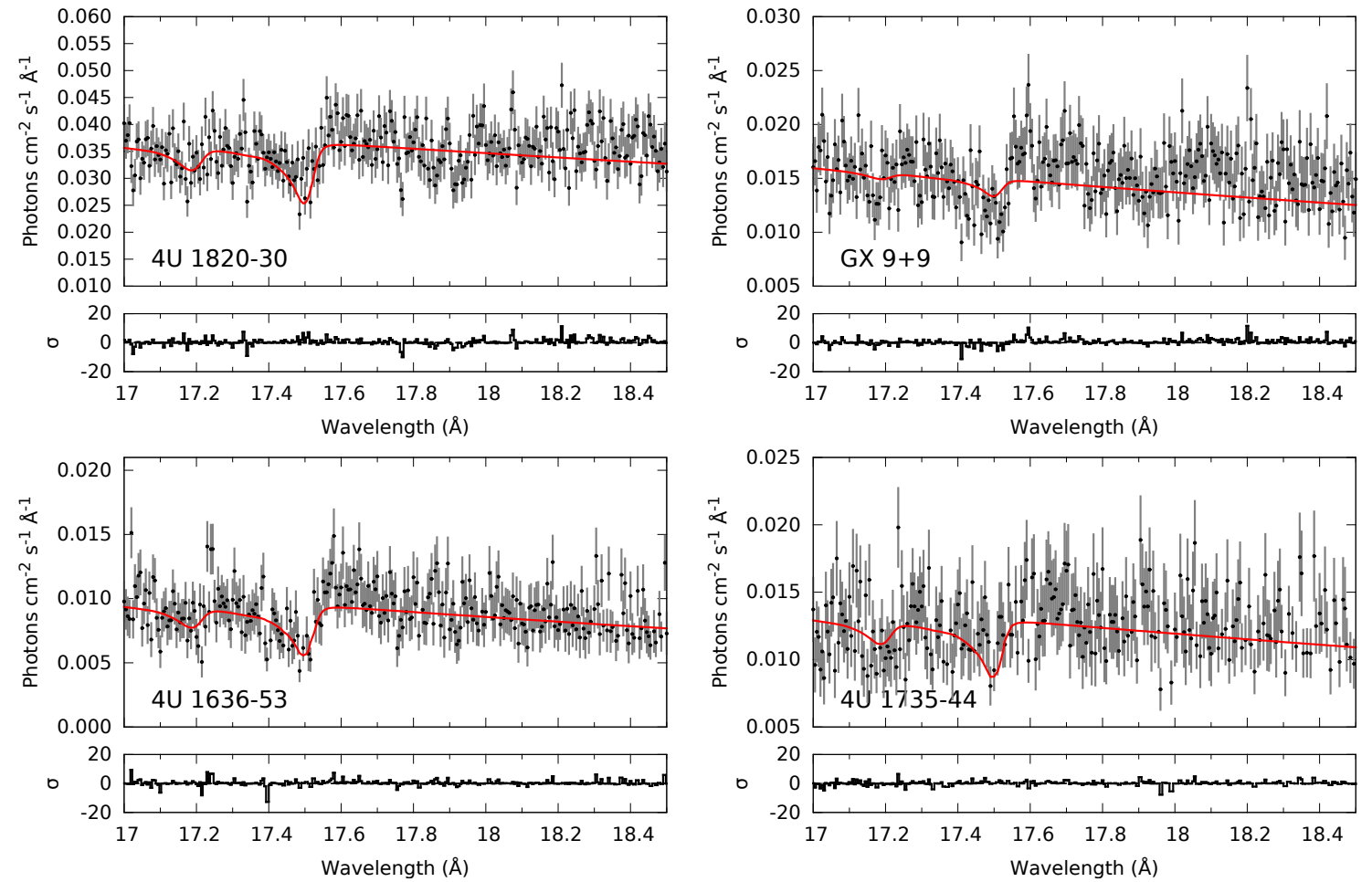

Figure 9. ISMabs best broadband fit (solid red lines) in the Fe L-edge region. For each source the observations have been combined for illustrative purposes. 
Table 5

H Column Densities $\left(10^{21} \mathrm{~cm}^{-2}\right)$

\begin{tabular}{lclll}
\hline \hline \multicolumn{1}{c}{ Source } & ISMabs & $21 \mathrm{~cm}^{a}$ & \multicolumn{1}{c}{ Juett $^{b}$} & \multicolumn{1}{c}{ Others } \\
\hline 4U 1636-53 & $4.16 \pm 0.42$ & 3.30 & $5.3_{-0.7}^{+2.1}$ & $3.9 \pm 1.2^{c}$ \\
4U 1735-44 & $1.88 \pm 0.48$ & 3.03 & $7 \pm 2^{c}$ & $3.0^{c}$ \\
4U 1820-30 & $0.92 \pm 0.16$ & 1.52 & $2.7_{-0.3}^{+0.4}$ & $0.78 \pm 0.03^{d}$ \\
GX 9+9 & $3.15 \pm 0.21$ & 2.10 & $4.5_{-1.2}^{+2.4}$ & \\
Cygnus X-1 & $6.23 \pm 0.14$ & 8.10 & $5.35 \pm 0.6$ & $6.6_{-0.3}^{+0.8} e$ \\
& & & & $5.4 \pm 0.4^{f}$ \\
Cygnus X-2 & $0.59 \pm 0.08$ & 2.20 & $2.3 \pm 0.5$ & $1.9 \pm 0.5^{g}$ \\
Sco X-1 & $1.10 \pm 0.30$ & 1.47 & & $1.33 \pm 0.02^{h}$ \\
& & & & $1.1 \pm 0.5^{i}$ \\
XTE J1817-330 & $0.38 \pm 0.20$ & 1.58 & &
\end{tabular}

\footnotetext{
a Dickey \& Lockman 1990.

b Juett et al. (2004).

c Cackett et al. (2009).

$\mathrm{d}$ Costantini et al. (2012).

e Makishima et al. 2008.

f Hanke et al. (2009).

$\mathrm{g}$ Costantini et al. (2005).

h García et al. (2011).

i Bradshaw et al. 2003).
}

by Juett et al. (2004). For an atomic element $X$, say, $N(X)=$ $N\left(X^{0}\right)+N\left(X^{+}\right)+N\left(X^{+2}\right)$. The respective column densities by Juett et al. (2004) were obtained through a fit of eight galactic sources which includes those of the present report except for Sco X-1 and XTE J1817-330. The average values for the oxygen column densities are $N(\mathrm{O})=(12.96 \pm 1.52) \times$ $10^{21} \mathrm{~cm}^{-2}$ (ISMabs) and $N(\mathrm{O})=(24.00 \pm 7.43) \times 10^{21} \mathrm{~cm}^{-2}$ (Juett et al. 2004). In the case of neon we obtained an average value $N(\mathrm{Ne})=(3.89 \pm 0.57) \times 10^{21} \mathrm{~cm}^{-2}($ I SMabs $)$ compared to $N(\mathrm{Ne})=(4.05 \pm 1.06) \times 10^{21} \mathrm{~cm}^{-2}$ (Juett et al. 2004). Lastly, for iron we estimated an average value of $N(\mathrm{Fe})=(5.85 \pm 1.12) \times 10^{21} \mathrm{~cm}^{-2}($ I SMabs $)$ compared to $N(\mathrm{Fe})=(7.55 \pm 1.76) \times 10^{21} \mathrm{~cm}^{-2}$ by Juett et al. $(2004)$.

From such data we have estimated the $N(\mathrm{O}) / N(\mathrm{Ne})$ and $N(\mathrm{Fe}) / N(\mathrm{Ne})$ column-density ratios derived from the ISMabs broadband fit. Neon is chosen as a reference since, as a noble gas, it is neither depleted to molecular nor to solid forms. The present average ratio is $N(\mathrm{O}) / N(\mathrm{Ne})=2.85 \pm$ 0.37 , while that derived by Juett et al. (2006) is $N(\mathrm{O}) / N(\mathrm{Ne})=$ $3.7 \pm 0.3$. Ratio differences may by due to systematic effects such as line saturation as quoted by Gatuzz et al. (2013) for the case of XTE J1817-330. The ratio derived by Juett et al. (2006) is higher than the present value; however, as discussed by these authors, the inclusion of black-hole systems (e.g. Cygnus $\mathrm{X}-1$ ) may modify results due to the presence of intrinsic emission or absorption features. We also obtain the average ratio $N(\mathrm{Fe}) / N(\mathrm{Ne})=0.12 \pm 0.02$ which is lower than solar, namely $N(\mathrm{Fe}) / N(\mathrm{Ne})=0.26$ (Grevesse \& Sauval 1998) and $N(\mathrm{Fe}) / N(\mathrm{Ne})=0.48$ (Asplund et al. 2005). This discrepancy may be due to either ISM Fe depletion or $\mathrm{Ne}$ overabundance. In fact, as previously discussed, we have found a $\mathrm{Ne}$ abundance greater than solar in the lines of sight toward 4U 1636-53, 4U 1735-44, GX 9+9, and XTE J1817-330. The $N(\mathrm{Fe}) / N(\mathrm{Ne})$ ratio by Juett et al. (2006) $(N(\mathrm{Fe}) / N(\mathrm{Ne})=0.15 \pm 0.01)$, on the other hand, is in a fair agreement with ISMabs. A further comparison of the iron and oxygen column densities finds the ISMabs ratio $N(\mathrm{Fe}) / N(\mathrm{O})=0.04 \pm 0.01$ in close agreement with the solar ratio of Grevesse \& Sauval (1998) $(\mathrm{Fe} / \mathrm{O}=0.04)$ but somewhat lower than that by Asplund et al. (2005) $(N(\mathrm{Fe}) / N(\mathrm{O})=$

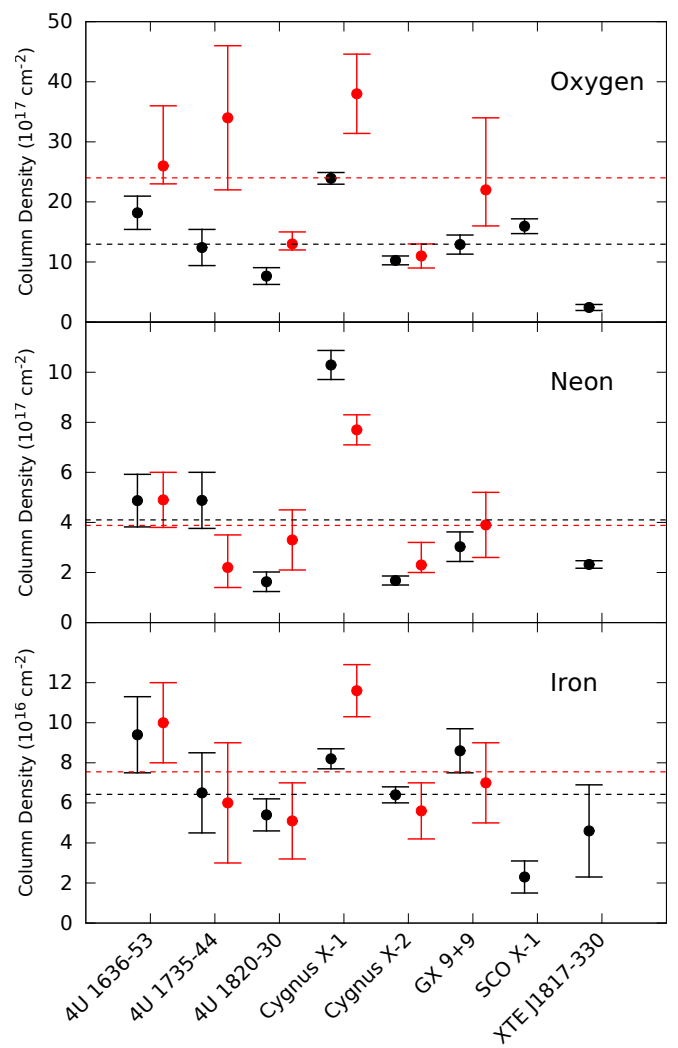

Figure 10. Comparison of the oxygen, neon and iron total column densities obtained from the ISMabs model (black lines) and those derived by Juett et al. 2004, 2006) (red lines). The average ratios are plotted with a dashed horizontal line.

0.06). Future analysis of the Fe K-edge absorption region would indeed help to constrain the ISM Fe abundance fractions in order to settle this issue.

The high-resolution X-ray spectra toward eight LMXBs have been analyzed. This study involved a broadband fit with ISMabs to constrain the hydrogen column density, finding good agreement with previous work. Then, a simultaneous fit of the neon- and iron-edge regions was performed for each source, where the resulting column densities indicate the presence of a lowly ionized gas along the different lines of sight.

We intend to carry out in the near future a more extensive analysis of ISM photoabsorption using all the grating spectra from X-ray binaries available from the XMM-Newton and Chandra archives. Our target is to create a library of column densities and abundances for all the detectable atomic species in these spectra. We believe that once the atomic $\mathrm{K}$ edges are fully analyzed and modeled, the search for molecular and dust spectral features can be pursued in earnest, a topic of current interest in ISM astrophysics. Finally, the present ISMabs model can be downloaded 9 for use in X-ray data analysis packages such as XSPEC (Arnaud 1996), ISIS (Houck \& Denicola 2000) and SHERPA (Freeman et al. 2001).

This work was supported by grant 15400673 of the Chandra Theory Program.

${ }^{9}$ http://heasarc.gsfc.nasa.gov/xanadu/xspec/models//ismabs.html 
Table 6

ISMabs Broadband Simultaneous Fit Parameters

\begin{tabular}{|c|c|c|c|c|c|c|c|c|c|}
\hline \multirow[t]{2}{*}{ Source } & \multicolumn{8}{|c|}{ ISMabs } & \multirow{2}{*}{$\begin{array}{c}\text { Statistics } \\
\chi^{2} / \text { dof }\end{array}$} \\
\hline & $N(\mathrm{H})$ & $N(\mathrm{O} \mathrm{I})$ & $N(\mathrm{O}$ II $)$ & $N(\mathrm{O}$ III $)$ & $N(\mathrm{Ne} \mathrm{I})$ & $N(\mathrm{Ne} \mathrm{II})$ & $N(\mathrm{Ne}$ III $)$ & $N(\mathrm{Fe})$ & \\
\hline 4U 1636-53 & $4.16 \pm 0.42$ & $17.43 \pm 2.53$ & $0.18 \pm 0.10$ & $0.58 \pm 0.14$ & $4.00 \pm 0.67$ & $0.78 \pm 0.37$ & $0.09 \pm 0.01$ & $0.94 \pm 0.19$ & $3411 / 4313$ \\
\hline $4 U$ 1735-44 & $1.88 \pm 0.48$ & $11.49 \pm 2.70$ & $0.43 \pm 0.20$ & $0.49 \pm 0.11$ & $3.93 \pm 0.75$ & $0.91 \pm 0.35$ & $0.04 \pm 0.02$ & $0.65 \pm 0.20$ & $1390 / 2293$ \\
\hline $4 U$ 1820-30 & $0.92 \pm 0.16$ & $6.99 \pm 0.93$ & $0.45 \pm 0.32$ & $0.22 \pm 0.15$ & $1.25 \pm 0.26$ & $0.33 \pm 0.11$ & $0.05 \pm 0.02$ & $0.54 \pm 0.08$ & $6605 / 7943$ \\
\hline GX 9+9 & $3.15 \pm 0.21$ & $12.30 \pm 1.12$ & $0.35 \pm 0.28$ & $0.24 \pm 0.19$ & $2.47 \pm 0.40$ & $0.51 \pm 0.16$ & $0.05 \pm 0.03$ & $0.86 \pm 0.11$ & $3492 / 3277$ \\
\hline Cygnus X-1 & $6.23 \pm 0.14$ & $23.38 \pm 0.72$ & $0.16 \pm 0.12$ & $0.38 \pm 0.13$ & $9.51 \pm 0.35$ & $0.64 \pm 0.18$ & $0.14 \pm 0.05$ & $0.82 \pm 0.05$ & $7156 / 4275$ \\
\hline Cygnus X-2 & $0.59 \pm 0.08$ & $9.52 \pm 0.45$ & $0.55 \pm 0.22$ & $0.20 \pm 0.07$ & $1.25 \pm 0.12$ & $0.39 \pm 0.05$ & $0.04 \pm 0.01$ & $0.64 \pm 0.04$ & $8986 / 8941$ \\
\hline Sco X-1 & $1.10 \pm 0.27$ & $15.78 \pm 1.19$ & $0.17 \pm 0.04$ & $0.00 \pm 0.00$ & (fixed) & (fixed) & (fixed) & $0.23 \pm 0.08$ & $1520 / 1378$ \\
\hline XTE J1817-330 & $0.38 \pm 0.20$ & $1.10 \pm 0.32$ & $0.87 \pm 0.11$ & $0.46 \pm 0.07$ & $1.56 \pm 0.10$ & $0.66 \pm 0.04$ & $0.10 \pm 0.01$ & $0.46 \pm 0.23$ & $16335 / 9885$ \\
\hline
\end{tabular}

Note. $-N(\mathrm{H})$ in units of $10^{21} \mathrm{~cm}^{-2}$. Metal column densities in units of $10^{17} \mathrm{~cm}^{-2}$.

\section{APPENDIX}

\section{ROLE OF IONIZATION BALANCE ON ABUNDANCES DETERMINATION}

Here we discuss the differences in fitting spectra with the ISMabs and warmabs models, the latter as implemented in previous work (Gatuzz et al.2014; Gatuzz et al.2013). An important complication affecting our modeling concerns the smooth background photoelectric absorption which cannot be identified uniquely by spectral features. This background is always present, and arises from hydrogen, helium, and valence-shell absorption by the same ions responsible for the edges and resonance absorption lines. Since all these mechanisms produce a smooth opacity, it is impossible to distinguish them uniquely. If the gas is assumed to be in ionization balance, then the relative columns of neutral H, He I, and He II are determined; if not, it is important to be aware of the degeneracy between the smooth absorption produced by $\mathrm{H}, \mathrm{He} \mathrm{I}$, and He II.

This degeneracy is illustrated graphically in Figure 11, which shows the $\chi^{2}$ contours of the fit to the XTE J1817-330 spectrum for various combinations of the $\mathrm{H}$ and He II column densities. The solid curves show contours of constant $\chi^{2}$ spaced at $\Delta \chi^{2}=100$. This clearly illustrates that the combinations of these variables lying along the diagonal are equivalent while those that fit the data have an almost exact inverse relationship. This obvious behavior must be understood and accounted for when deriving abundances relative to $\mathrm{H}$ or $\mathrm{He}$ in X-ray spectral fitting. In the results reported in the main body of this paper, which are based on ISMabs, we have assumed that all the smooth opacity in the X-ray band comes from $\mathrm{H}+\mathrm{He} \mathrm{I}$ and that the He II column densities are negligible.

The warmabs model includes the results of a photoionization equilibrium calculation, and therefore, the $\mathrm{H}$ and He II column densities are tied together. The value taken from the warmabs fit of the XTE J1817-330 spectrum is shown with a dot in Figure 11. This corresponds to an ionization parameter of $\log \xi=-2.5$ for which the column density of neutral $\mathrm{H}+\mathrm{He} \mathrm{I}$ is 10.8 times that of He II; i.e., the equilibrium assumption removes the degeneracy between the two variables.

A quantity of interest associated to the fitting of interstellar X-ray absorption spectra is the abundance of elements such as oxygen and neon relative to hydrogen. If the smooth background absorption in the X-ray band were solely due to neutral $\mathrm{H}$, then the $\mathrm{H}$ and $\mathrm{O}$ or Ne column densities derived from the fits could be used to derive the relevant abundance ratios by simply taking

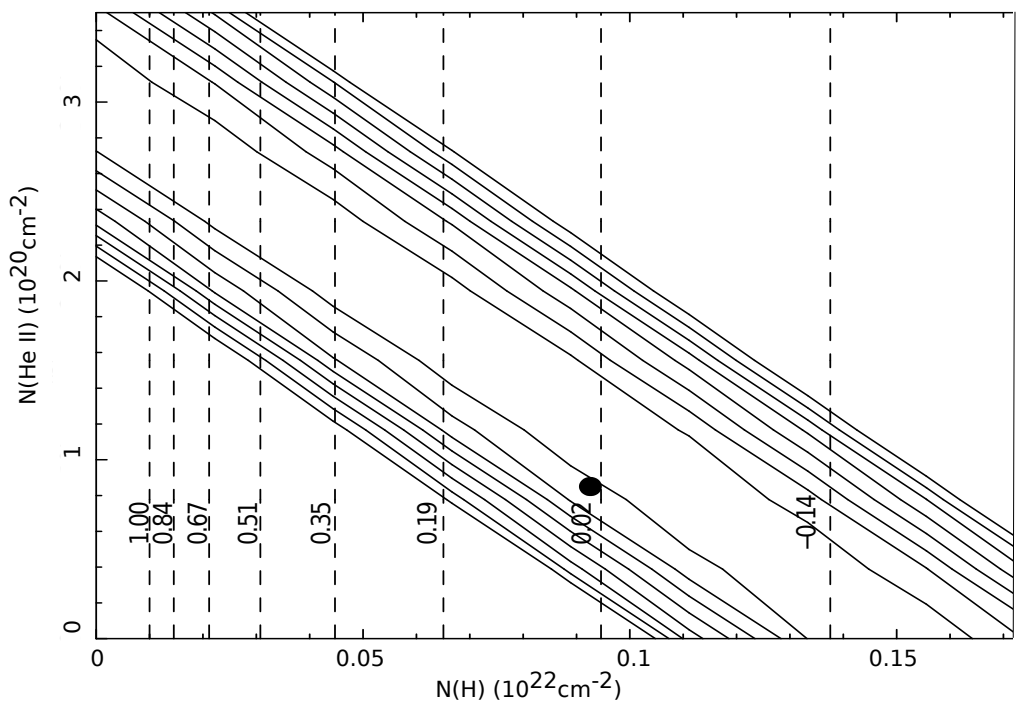

Figure 11. Constant $\chi^{2}$ contours for the broadband fits of the XTE J1817-304 spectrum. The axes correspond to He II and H column densities. Vertical dashed lines show the degeneracy between these two quantities since the neutral H column cannot be uniquely determined. The adopted warmabs best-fit value is denoted with a black dot. 


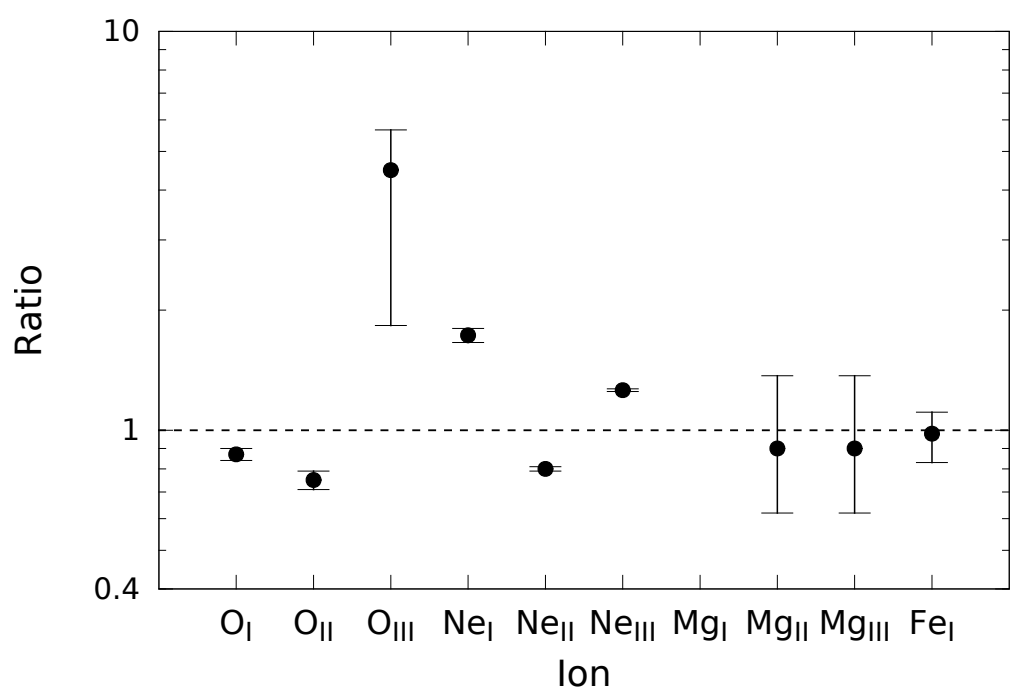

Figure 12. Ion column densities obtained from the fit to the XTE J1817-304 spectrum. Each point corresponds to the ISMabs/warmabs ratio.

column-density ratios. The degeneracy between $\mathrm{He}$ II and $\mathrm{H}$ complicates this procedure since the total $\mathrm{H}$ column density cannot be uniquely determined. This is shown graphically in Figure 11 with the vertical dashed curves which are curves of constant $\mathrm{O}$ abundance relative to $\mathrm{H}$ for the fit to the spectra of XTE J1817-330. The curves are labeled according to the conventional notation: $[\mathrm{O}]=0$ corresponds to the solar oxygen abundance (Grevesse \& Sauval 1998), [O] $=1$ to $10 \times$ solar, etc. This shows that, although acceptable fits can be obtained for any value of $N(\mathrm{H})$ in the range up to $2 \times 10^{21} \mathrm{~cm}^{-2}$, the inferred abundance of $\mathrm{O}$ relative to $\mathrm{H}$ can take any value from 0.75 solar to 10 times solar.

Finally, we mention the unavoidable limitation that different numerical model implementations, even if they are based on the same atomic data, inevitably differ due to numerical details. For example, calculations in ISMabs use cross sections tabulated on a grid that is finer than most instrumental grids, but which inevitably requires interpolation or smoothing to produce a model for XSPEC. warmabs, on the other hand, adopts such tabulations for some cross sections while for others analytic expressions are employed. Examples of the latter are the all-important cross sections for neutral $\mathrm{O}, \mathrm{Ne}$, and $\mathrm{Mg}$ of Gorczyca et al. (2013). Also, is important to note that the Fe cross-sections are different between both models, I SMabs uses Kortright \& Kim (2000) cross-section while warmabs uses Verner \& Yakovlev (1995) cross section. Small numerical differences in the cross section or opacity are exponentially amplified in the transmission coefficient which is most closely related to the observed spectrum. Thus, in spite of efforts to minimize numerical differences between codes, they are unavoidable at some level and it is useful to attempt to quantify them.

To summarize, we identify the three principal issues that affect the quantitative results of fitting to interstellar absorption and model comparisons: (i) the importance of ions and whether they are treated in equilibrium ratios or not; (ii) the ambiguity associated to the smooth background; and (iii) numerical implementation issues. We now proceed to illustrate them with quantitative examples.

In Figure 12 we show a comparison of the ion column densities derived from fitting to XTE J1817-330 with warmabs and ISMabs. In the case of warmabs, we adopt two ionization components with $\log \xi=-5$ and $\log \xi=-2.5$, both with a column density of $10^{21} \mathrm{~cm}^{-2}$. The elemental abundances for the two components are allowed to vary freely in order to obtain the best fit. The four observations of this source are fitted simultaneously, and all the absorption parameters (i.e. the warmabs ionization parameters, column densities, elemental abundances, turbulent velocities, and redshifts) are forced to be the same for all the different observations. The continuum is taken to be the sum of a black body and a power law, and the parameters of these components are allowed to differ between the various observations to reflect source variability. The best fit has a $\chi^{2} / \nu=28325.47 / 15183=1.866$. warmabs can output a list of ion column densities (even though these are not free parameters), and in Figure 12 we show a comparison of the warmabs ion column densities with those from an ISMabs fit. In the latter the $\mathrm{H}$ and He II column densities are fixed at the values taken from the warmabs fit. We take the column densities from all the ions from the warmabs best fit and put them into ISMabs. This includes the H, He I, and He II column densities. Using the same continuum parameters we obtain $\chi^{2} / \nu=29876 / 15183=1.968$. This $\chi^{2}$ difference is likely to be numerical since all other parameters of the two fits are identical. We then allow the ion column densities in ISMabs and the continuum parameters to vary freely in order to obtain the best fit. In doing so we do not allow the $\mathrm{H}$, He I, and He II column densities to vary, and we preserve the restriction that the absorption model is the same for all observations. This procedure produces a fit with $\chi^{2} / \nu=27203 / 15153=1.795$.

Figure 12 shows the ratio of the column densities derived from these two different methods. This represents both an astrophysical experiment on whether the ratios show photoionization equilibrium, and in some sense, a test of model accuracy (note that the Si and S column densities are negligible in both fits, so they are omitted). The two fits agree to within the error bars for most ions, a notable exception being O III for which the warmabs model predicts a significantly lower column density than ISMabs. This indicates the existence of a component in the absorbing gas toward this source which is more highly ionized than the equilibrium gas that can actually fit most neutrals and singly ionized species. Oxygen shows such an effect more clearly than 


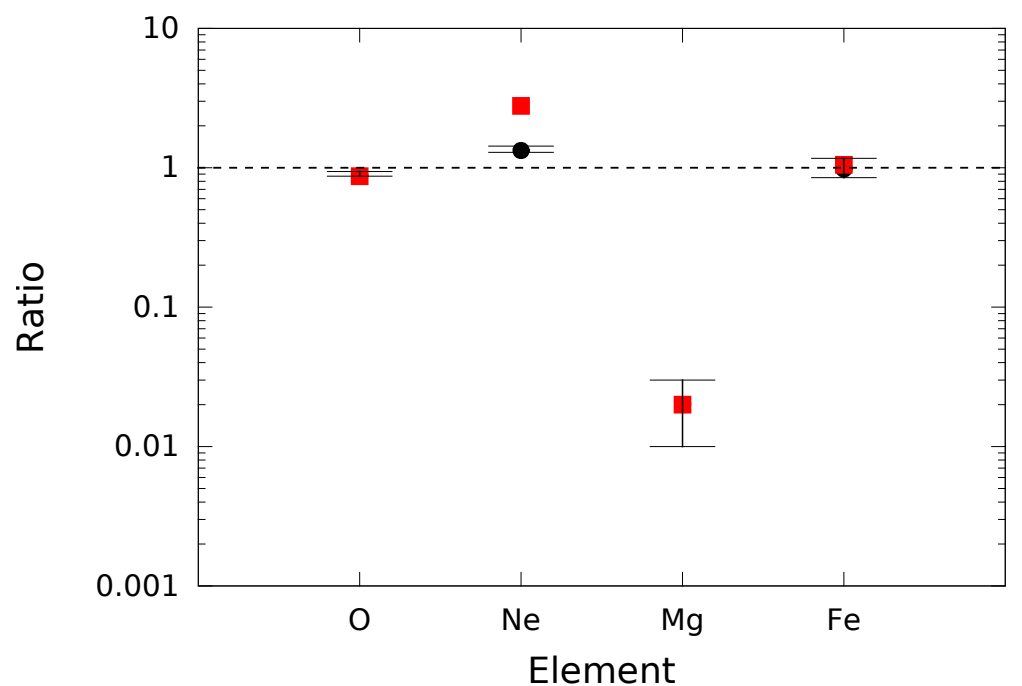

Figure 13. Abundances for various atomic elements obtained from the fit to XTE J1817-304. Black circles correspond to the ISMabs/warmabs abundance ratios while the red squares indicate the ratios of ISMabs to the solar abundances of Anders \& Grevesse (1989).

other elements due to its relatively high abundance.

A further step in exploring the effects of ionization equilibrium in absorption fitting is to sum the ion column densities derived from the fits with and without equilibrium (i.e. using warmabs or ISMabs) to get elemental abundances. Results are shown in Figure 13 where the ratio of elemental abundances are obtained by summing up the ISMabs ion column densities to the elemental abundances obtained from the warmabs column densities. The abundances shown here for ISMabs make use of the $\mathrm{H}$ and $\mathrm{He}$ II column densities derived by warmabs. Also shown are the ratio of the ISMabs abundances to the values in the compilation by Grevesse \& Sauval (1998). This shows that, in spite of the disagreement for O III, both fitting methods yield abundances that are consistent with each other and with the compilation. However, the Ne abundance in this source is an exception; ISMabs manifests a tendency to produce larger abundances since it is capable of accounting for ion absorption that is not tied up to the ionization equilibrium assumption. 


\section{REFERENCES}

Anders, E., \& Ebihara, M. 1982, GeCoA, 46, 2363

Anders, E., \& Grevesse, N. 1989, GeCoA, 53, 197

Arnaud, K. A. 1996, in ASP Conf. Ser. 101, Astronomical Data Analysis Software and Systems V, ed. G. H. Jacoby, \& J. Barnes (San Francisco, CA: ASP), 17

Asplund, M., Grevesse, N., \& Sauval, A. J. 2005, in ASP Conf. Ser. 336, Cosmic Abundances as Records of Stellar Evolution and Nucleosynthesis, ed. T. G.

Barnes III, \& F. N. Bash (San Francisco, CA: ASP), 25

Balucinska-Church, M., \& McCammon, D. 1992, ApJ, 400, 699

Bethe, H. A., \& Salpeter, E. E. 1957, Quantum Mechanics of One- and Two-Electron Atoms, ed. Springer, (Berlin)

Bradshaw, C. F., Fomalont, E. B., \& Geldzahler, B. J. 1999, ApJ, 512, L121

Bradshaw, C. F., Geldzahler, B. J., \& Fomalont, E. B. 2003, ApJ, 592, 486

Brandt, S., Castro-Tirado, A. J., Lund, N., Dremin, V., Lapshov, I., \& Syunyaev, R. 1992, A\&A, 262, L15

Cackett, E. M., et al. 2009, ApJ, 690, 1847

Costantini, E., Freyberg, M. J., \& Predehl, P. 2005, A\&A, 444, 187

Costantini, E., et al. 2012, A\&A, 539, A32

Dickey, J. M., \& Lockman, F. J. 1990, ARA\&A, 28, 215

Dwek, E., \& Arendt, R. G. 1992, ARA\&A, 30, 11

Freeman, P., Doe, S., \& Siemiginowska, A. 2001, in Society of Photo-Optical Instrumentation Engineers (SPIE) Conference Series, Vol. 4477, Astronomical Data Analysis, ed. J.-L. Starck \& F. D. Murtagh, 76-87

Galloway, D. K., Muno, M. P., Hartman, J. M., Psaltis, D., \& Chakrabarty, D. 2008, ApJS, 179, 360

García, J., Mendoza, C., Bautista, M. A., Gorczyca, T. W., Kallman, T. R., \& Palmeri, P. 2005, ApJS, 158, 68

García, J., Ramírez, J. M., Kallman, T. R., Witthoeft, M., Bautista, M. A., Mendoza, C., Palmeri, P., \& Quinet, P. 2011, ApJ, 731, L15

García, J., et al. 2009, ApJS, 185, 477

Gatuzz, E., GarcÃna, J., Mendoza, C., Kallman, T. R., Bautista, M. A., \& Gorczyca, T. W. 2014, ApJ, 790, 131

Gatuzz, E., et al. 2013, ApJ, 768, 60

Gorczyca, T. W. 2000, PhRvA, 61, 024702

Gorczyca, T. W., et al. 2013, ApJ, 779, 78

Grevesse, N., \& Sauval, A. J. 1998, SSRv, 85, 161

Hanke, M., Wilms, J., Nowak, M. A., Pottschmidt, K., Schulz, N. S., \& Lee, J. C. 2009, ApJ, 690, 330

Hasoğlu, M. F., Abdel-Naby, S. A., Gatuzz, E., García, J., Kallman, T. R., Mendoza, C., \& Gorczyca, T. W. 2014, The Astrophysical Journal Supplement Series, 214,8

Henke, B. L., Lee, P., Tanaka, T. J., Shimabukuro, R. L., \& Fujikawa, B. K. 1982, ADNDT, 27, 1

Houck, J. C., \& Denicola, L. A. 2000, in Astronomical Society of the Pacific Conference Series, Vol. 216, Astronomical Data Analysis Software and Systems

IX, ed. N. Manset, C. Veillet, \& D. Crabtree, 591

Jenkins, E. B. 2005, HiA, 13, 802

Juett, A. M., Schulz, N. S., \& Chakrabarty, D. 2004, ApJ, 612, 308

Juett, A. M., Schulz, N. S., Chakrabarty, D., \& Gorczyca, T. W. 2006, ApJ, 648, 1066

Kortright, J. B., \& Kim, S.-K. 2000, PhRvB, 62, 12216

Lee, J. C., \& Ravel, B. 2005, ApJ, 622, 970

Lee, J. C., Xiang, J., Ravel, B., Kortright, J. \& Flanagan, K. 2009, ApJ, 702, 970

Liao, J.-Y., Zhang, S.-N., \& Yao, Y. 2013, ApJ, 774, 116

Liu, Q. Z., van Paradijs, J., \& van den Heuvel, E. P. J. 2007, A\&A, 469, 807

Lodders, K. 2003, ApJ, 591, 1220

Makishima, K., et al. 2008, PASJ, 60, 585

Massey, P., Johnson, K. E., \& Degioia-Eastwood, K. 1995, ApJ, 454, 151

Migliari, S., Fender, R. P., Rupen, M., Wachter, S., Jonker, P. G., Homan, J., \& van der Klis, M. 2004, MNRAS, 351, 186

Miller, J. M., Cackett, E. M., \& Reis, R. C. 2009, ApJ, 707, L77

Morrison, R., \& McCammon, D. 1983, ApJ, 270, 119

Orosz, J. A., \& Kuulkers, E. 1999, MNRAS, 305, 132

Pinto, C., Kaastra, J. S., Costantini, E., \& de Vries, C. 2013, A\&A, 551, A25

Pinto, C., Kaastra, J. S., Costantini, E., \& Verbunt, F. 2010, A\&A, 521, A79

Sala, G., \& Greiner, J. 2006, ATel, 791, 1

Stolte, W. C., et al. 1997, JPhB, 30, 4489

Verner, D. A., \& Yakovlev, D. G. 1995, A\&AS, 109, 125

Wilms, J., Allen, A., \& McCray, R. 2000, ApJ, 542, 914

Witthoeft, M. C., Bautista, M. A., Mendoza, C., Kallman, T. R., Palmeri, P., \& Quinet, P. 2009, ApJS, 182, 127

Witthoeft, M. C., García, J., Kallman, T. R., Bautista, M. A., Mendoza, C., Palmeri, P., \& Quinet, P. 2011, ApJS, 192, 7

Yao, Y., Schulz, N. S., Gu, M. F., Nowak, M. A., \& Canizares, C. R. 2009, ApJ, 696, 1418

Zdziarski, A. A., Gierliński, M., Mikołajewska, J., Wardziński, G., Smith, D. M., Harmon, B. A., \& Kitamoto, S. 2004, MNRAS, 351,791 